\title{
Surface wave effects on long range IR imaging in the marine surface layer
}

\author{
M. J. Francius, G. J. Kunz, and A. M. J. van Eijk, \\ TNO Defense, Security and Safety, P.O. Box 96864, 2509 JG The Hague, The Netherlands, Tel: +31 \\ 70374 0865, Fax: +31 70374 0654, Email: marc.francius@tno.nl
}

\begin{abstract}
The quality of long range infrared (IR) imaging depends on the effects of atmospheric refraction and other pathintegrated effects (e.g., transmission losses, scintillation and blurring), which are strongly related to the prevailing meteorological conditions. EOSTAR is a PC based computer program to quantify these strong nonlinear effects in the marine atmospheric surface layer and to present a spectrally resolved target image influenced by atmospheric effects using ray tracing techniques for the individual camera pixels. Presently, the propagation is predicted with bulk atmospheric models and the sea surface is idealized by steady regular periodic Stokes' waves. Dynamical wind-waves interactions are not taken into account in this approach, although they may strongly modify the refractive index in the near-surface layer. Nonetheless, the inclusion of the sea surface in the ray tracer module already has a great impact on the near-surface grazing rays and thus influences the images especially in situations of super refraction and mirage. This work aims at improving the description of the sea surface in EOSTAR taking into account the non-uniformity of spatially resolved wind-generated waves and swell. A new surface module is developed to model surface wind-waves and swell in EOSTAR on the basis of meteorological observations and spectral wave modeling. Effects due to these new surfaces will be analyzed and presented.
\end{abstract}

Keywords: ray tracer, atmospheric refraction, mirage, surface waves

\section{INTRODUCTION}

The EOSTAR model assesses the effects of the marine atmosphere on the quality of long-range infrared (IR) imaging. The dominant atmospheric effects, atmospheric refraction and other path-integrated effects (e.g., transmission losses, scintillation and blurring), are strongly related to the prevailing meteorological conditions and can be parameterized through semi-empirical models. Then by using ray tracing techniques for the individual camera pixels, it is possible to analyze the influence of atmospheric effects on the spectrally resolved target images product of EOSTAR (Kunz et al., 2004). In this work, we will pay particular attention to another factor in the marine environment, i.e., the effects of surface waves on the quality of long-range IR imaging. Surface waves have significant effects on the observation of long distant targets, especially under unstable conditions. In those cases, the overlaying air is colder than the sea surface, resulting in a local positive gradient of refractivity. As a result, ray trajectories starting at a negative elevation angle will be refracted upwards and, generally, this effect will be stronger when the initial angle of the ray trajectory is more negative. This will lead to situations that different camera pixels are observing the same volume, and thus cause mirage images of targets in that volume. The shortest distance at which the mirage is observed (minimum mirage range or MMR) is determined by the ray that passes closest over the wave surface. Hence, the MMR depends strongly on the (instantaneous) height of the waves and the MMR decreases as wave height increases. This work is a first step towards implementing the statistical properties of the wavy surface in a ray-tracing program and analyzing geometrical micrometeorological effects. Two methods for a probabilistic description of the maximum wave height will be discussed and the sensitivity of EOSTAR calculations on the wave height will be investigated.

Atmospheric Optical Modeling, Measurement, and Simulation, edited by Stephen M. Doss-Hammel, Anton Kohnle, Proc. of SPIE Vol. 5891 (SPIE, Bellingham, WA, 2005) · 0277-786X/05/\$15 · doi: 10.1117/12.619953 


\section{EOSTAR}

EOSTAR (Kunz et al., 2004) is a collection of interacting computer programs to predict refraction, turbulence and transmission in the marine surface layer. This program uses Monin-Obukhov similarity theory to calculate vertical profiles of temperature, humidity, refractivity and structure function parameter of air from standard meteorological input data. Molecular atmospheric transmission and path radiation are calculated using MODTRAN, whereas the Advanced Navy Aerosol Model (ANAM, Van Eijk et al., 2002) is used for aerosol absorption and scattering. Infrared sky and sea surface backgrounds are calculated using a separate module that is based on experimental data (Marine Infrared Background Simulator MIBS, Schwering 1996). Alternatively, MODTRAN can be used. Ray tracing techniques are used to calculate the observation paths of the individual camera pixels, taking into account effects of refraction, turbulence and transmission. Target infrared signatures are synthesized using segment decomposition, and subsequently taking into account the physical properties of each segment, the spectra of the incoming radiation, and the spectral reflection properties of each segment. The overall signature is calculated with a heat flux balance code, the Electro-Optical Signature Module EOSM (Neele, 2005). The final result of EOSTAR is the synthetic sensor image of the target in the specified marine environment.

In its simplest approach, the ray trajectories module ignores the presence of surface waves and places the surface at mean sea level. This is allowed for conditions of super refraction in which the sea surface is colder than the over laying air. However, as mentioned in the introduction, this approach is not justified for near-neutral and unstable conditions. In those cases 'rays' initially starting at a negative elevation angle are bent upwards due to the positive vertical gradient of the refractivity. Whether such a ray is bent upwards to the extent that it will not hit the water surface depends critically on the initial direction of the ray, the height of the surface and, of course, the profile of the refractivity. As a consequence, the maximum observation range (also referred to as maximum intervision range or MIVR), the MMR (see introduction) and the length of the mirage zone depend on these parameters. To account for these effects, EOSTAR can simulate IR-imagery using a modest wave module. This module uses an $8^{\text {th }}$ order Stokes approximation to calculate the mean height of the waves, and EOSTAR subsequently places a flat surface at this mean wave height. In the following section, a more elaborate wave module for EOSTAR will be discussed.

\section{SURFACE WAVE MODEL}

\subsection{Background}

The EOSTAR model suite includes a simple wave module, which calculates the height of wind-driven surface waves based on Stokes theory for deep water waves. In the EOSTAR application, the geometrical characteristics of the surface waves are determined from the input wind speed $U_{z}$ at a reference height, usually $\mathrm{z}=10 \mathrm{~m}$. The surface wave field is modeled as a nonlinear sinusoidal wave train (EOSTAR uses an $8^{\text {th }}$ order Stokes wave) that depends only on two constants, the inverse wave age $U_{10} / C_{P}$ and the wave steepness $h$. It is assumed that for open ocean conditions, with fully developed waves, the parameters $U_{10} / C_{P}$ and $h$ take the values 0.85 and 0.135 , respectively:

$$
U_{10} / C_{p}=0.85, \quad h=H / \lambda_{p}=0.135
$$

The constants are related by the nonlinear dispersion relation

$$
2 \pi / \lambda_{p}=\frac{g}{C_{p}^{2}} \cdot f a c(h)
$$

In the above equations, parameter $C_{p}$ is the wave celerity, $H$ is the wave height and $\lambda_{\mathrm{p}}$ the wavelength of the Stokes wave. The term $f a c(h)$ accounts for nonlinear corrections (see, e.g., Geernaert, 1990).

However, there are several limitations to the use of Stokes wave formalism in EOSTAR. The first limitation concerns the relation between wave height and wind speed. While equation (1a) formally allows using $U_{10}$ as a driving parameter to assess $C_{p}$, the Stokes wave formalism is not suitable for describing wind-generated waves. The Stokes wave solution refers to a particular class of nonlinear surface wave motion that is not forced by the wind, such as swell waves that are 
generated by distant depression systems. In such a case, the local wind speed $U_{10}$ has little, if any, relation with the observed wave heights and should thus not be used as driving parameter. Furthermore, the value of $U_{10} / C_{P}$ for these swell waves is typically in the range $0.01-0.2$, whereas EOSTAR currently uses a value of 0.85 . The second limitation of the Stokes wave module lies in its idealized form. It is obvious that the Stokes wave field yields an idealized representation of the sea surface, since all the crests are located at exactly the same height. While this can be an acceptable simplification for swell waves, it fails for wind-generated waves that are randomly varying in time (at a given location) or in space (at a given time). This implies that an alternative wave module is required for wind-generated waves. Moreover, if we accept that the crest height varies in time or space, it is not sufficient to only know the average height of the crests when evaluating the cut-off height of ray trajectories. Instead, the statistics (probability of encountering a particular crest height) are required.

For these reasons, the present paper discusses a more general wave module, which allows to make stochastic predictions of the individual wave crests for any given sea state. The term sea state in this paper refers to a condition in which the statistical properties of the sea surface are quasi-stationary for a duration of $O(1 \mathrm{~h})$ and invariant over distances of $O(1$ $10 \mathrm{~km})$. The underlying assumption is that the waves are considered as a weakly steady-state homogeneous random process, thus ergodic. Details of stochastic analysis of random ocean waves can be found in several textbooks, for instance, Kinsman (1965) and Ochi (1990). For the present work it is sufficient to know that the sea-state can be described by a wave spectrum; that is, the power spectral density function of the vertical surface displacement $\eta(\mathbf{x}, t)$. This can be derived using the Wierner-Kintchine theorem, which starts with the covariance function $R(\boldsymbol{r}, t)$ defined as:

$$
R(\mathbf{r}, t)=<\eta\left(\mathbf{x}_{\mathbf{o}}, t_{0}\right) \eta\left(\mathbf{x}_{\mathbf{o}}+\mathbf{r}, t_{0}+t\right)>
$$

for any given position $\mathbf{x}_{\mathbf{0}}$ and time $t_{0}$, and space and time lags $\boldsymbol{r}$ and $t$, respectively. Here, the angle brackets represent the ensemble average operator. The wave spectrum $\Psi(\mathbf{k}, \omega)$ is just the Fourier transform of the covariance function given by equation (3),

$$
\Psi(\mathbf{k}, \omega)=(2 \pi)^{-3} \int R(\mathbf{r}, t) e^{-i(\mathbf{k} \cdot \mathbf{r}-\omega t)} \mathrm{d} \mathbf{r} \mathrm{d} t
$$

where $\mathbf{k}$ is the wave number vector and $\omega$ the wave frequency. By integrating the wave spectrum with respect to wavenumber $\mathbf{k}$, we obtain the frequency spectrum

$$
S(\omega)=\int \Psi(\mathbf{k}, \omega) \mathrm{d} \mathbf{k}
$$

which is an important quantity, because it can be experimentally obtained from a time history of wave records at a single point. The peak wavelength $\lambda_{p}$, peak period $T_{p}$ and peak frequency $\omega_{p}$ of the spectrum are evaluated at the maximum of $S(\omega)$. The frequency spectrum itself can be integrated to yield the $n$th spectral moments $m_{n}$ :

$$
m_{n}=\int \omega^{n} S(\omega) \mathrm{d} \omega \quad \mathrm{n}=0,1,2, \ldots
$$

As we will see later, these spectral moments form the basis of all expressions on wave statistics. They also determine basic parameters describing the sea state, such as the significant wave height $H_{s}$, the mean zero-crossing period $T_{z}$ and the significant steepness $s$ according to:

$$
\begin{aligned}
& H_{s}=4 \sqrt{m_{0}}=4 \sigma \\
& T_{z}=2 \pi \sqrt{m_{0} / m_{2}} \\
& s=\pi H_{s} / \lambda_{p}
\end{aligned}
$$

Here, $\sigma$ denotes the standard deviation $\left\langle\eta^{2}\right\rangle^{1 / 2}$ of the surface displacement at one point. 


\subsection{Probabilistic models for wave crest height}

For almost all sea states, the action of the wind results in a rather broad spectrum $S(\omega)$, ranging from long gravity waves with wavelengths up to several hundreds of meters to short gravity and capillary waves with wavelengths down to centimeters. The simplest and the most natural representation of the random sea surface for a given sea-state would thus be a linear superposition of many wave harmonics with random amplitude and phase that fulfill the dispersion relation of surface gravity waves. A mathematical representation of the surface displacement as a function of time at a given location based on this linear superposition concept can be written as:

$$
\eta(t)=\sum_{n=1}^{\infty} a_{n}(t) \cos \left(\omega_{n} t-\varphi_{n}\right)
$$

where $a_{n}(t)$ denotes the different random amplitudes of simple harmonic waves, coming from various directions, with random frequencies $\omega_{\mathrm{n}}$ and randomly phases $\varphi_{\mathrm{n}}$ uniformly distributed over $(-\pi, \pi)$. By invoking the well known Central Limit Theorem and assuming statistical independence of the wave amplitude and phase components, it can be shown that the sea surface displacement modeled by equation (7) must necessarily be a normal (Gaussian) process with zero mean and variance $\sigma^{2}$. The probability density function of the surface displacement is given by

$$
p(\eta)=\frac{1}{\sqrt{2 \pi \sigma^{2}}} e^{-\frac{1}{2}\left(\frac{\eta}{\sigma}\right)^{2}}
$$

where $p(\eta) \mathrm{d} \eta$ is the probability that the sea surface displacement occurs between heights $\eta$ and $\eta+\mathrm{d} \eta$. The mathematical derivation of this function can be found in the papers by Rice (1944) or Longuet-Higgins (1957). In general, the Gaussian property depends on sea severity (significant wave height) and water depth. For sufficiently deep-water waves, this assumption can be used irrespective of the sea severity and is related to the strength and duration of the wind. Waves in shallow water can also follow the Gaussian distribution laws when the sea severity is very mild. However, shallow water waves are usually more nonlinear than deep water waves, and the sea surface displacement often has non-Gaussian statistics in moderate to high wind conditions or in the presence of "shoaling" swell waves like in coastal areas.

In the statistical approach, other random wave parameters than the surface displacement can be of interest such as, for instance, wave height (defined as the crest-to-trough height) and wave period. For our purposes, we are interested in the statistics of crest height only. A problem arises here, since it is well known that the Gaussian probability density function (8) generally overestimates the probability of occurrence of large surface displacement. Given that larger wave-crest heights are of particular importance in the context of this study, we will not use equation (7) directly. Instead, we will focus on dedicated models for crest statistics.

The simplest models for the crest statistics of a "Gaussian" sea-state are usually derived under the narrow-band assumption. Under this assumption the wave spectrum is assumed to be sharply concentrated near a certain "peak" frequency $\omega_{p}$. This implies that the wave displacement in equation (7) can be rewritten as:

$$
\eta(t)=A(t) \cos \left(\omega_{p} t+\Phi(t)\right)
$$

where the wave amplitude $A(t)$ (height of surface above or below mean sea level) and the total phase $\Phi(t)$ are slowly varying functions of time. They are given by the relations:

$$
\begin{aligned}
& A(t)=\sqrt{A_{c}^{2}+A_{s}^{2}} \\
& \Phi(t)=\tan ^{-1}\left(A_{s} / A_{c}\right)
\end{aligned}
$$


in which

$$
\left.\begin{array}{l}
A_{c}=A \cos (\Phi)=\sum_{n=1}^{\infty} a_{n}(t) \cos \left(\left(\omega_{n}-\omega_{p}\right) t-\varphi_{n}\right) \\
A_{s}=A \sin (\Phi)=\sum_{n=1}^{\infty} a_{n}(t) \sin \left(\left(\omega_{n}-\omega_{p}\right) t-\varphi_{n}\right)
\end{array}\right\}
$$

are the crest and trough amplitudes (with respect to mean sea level), respectively. Under the narrow band assumption, the crest and trough amplitudes are slowly varying functions of time. When the temporal modulations of amplitude and frequency are constant, equation (9) reduces to a first order Stokes wave model. The $1^{\text {st }}$ order Stokes wave can thus be viewed as asymptotic narrow banded deterministic model. Higher order Stokes waves can be derived from equation (7). This requires that $\omega_{n}=n \omega_{p}$ and that some ordered "nonlinear" relation holds between the amplitude coefficients $\mathrm{a}_{\mathrm{n}}$.

Under the narrow band assumption, the positive and negative maximum excursions of the surface are almost equal to each other, which implies that the wave amplitude $A(t)$, which is also called the wave envelope, can be used directly to study the wave crest statistics. Longuet-Higgins (1957) has shown that the phase $\Phi(t)$ is uniformly distributed over $(-\pi$, $\pi$ ) and that the wave amplitude $A(t)$ follows the Rayleigh probability law. In that case, the probability density function (pdf) of the wave amplitude may be written in the general form:

$$
p_{A}(A)=\frac{2 A}{R} e^{-A^{2} / R}
$$

where $R=2 m_{0}$ is the parameter of the Rayleigh distribution and $m_{0}=\left\langle\eta^{2}\right\rangle$ is the $0^{\text {th }}$ spectral moment (cf. equation (6)). Using the dimensionless variable $\xi=A / m_{0}{ }^{l / 2}$, the (Rayleigh) probability density function (13) becomes in dimensionless form:

$$
f_{A}(\xi)=\xi e^{-\xi^{2} / 2}
$$

The expected mean value and variance of the wave amplitude can be obtained as:

$$
\left.\begin{array}{l}
\langle A\rangle=A_{\text {mean }}=\sqrt{\pi R / 4} \\
\left\langle\left(A-A_{\text {mean }}\right)^{2}\right\rangle=A_{\text {std }}^{2}=(1-\pi / 4) R
\end{array}\right\}
$$

The probability of occurrence of wave amplitude higher than a certain height $\zeta$ is defined as:

$$
\operatorname{Pr}\{A>\zeta\}=1-F_{A}(\zeta)
$$

where the cumulative distribution function $(\mathrm{cdf}) F_{A}(\zeta)$ is given by

$$
F_{A}(\zeta)=e^{-\frac{\zeta^{2}}{R}}
$$

Unfortunately, the narrow-band assumption resulting in equations (9)-(17) is not representative of all sea-state conditions. The profile of waves, in particular those observed in moderate to high wind conditions or with multi-modal seas (when wind waves and swell are both present), is much more irregular. It has local positive maxima and minima as well as local negative ones, and the waves occur in successive groups of higher and lower waves. However, it is still possible to use the concept of wave envelope $A(t)$, using relations (10) and (11), even when the spectrum is not narrow banded and the concept of wave amplitude can not be as clearly defined as in (9). The difference is that for non-narrowbanded spectra the positive maxima of the wave envelope do not necessarily coincide with the crest of the waves, and this more so when the spectrum is broader. Therefore, the use of wave envelope statistics is less suitable in the case of 
arbitrary wave spectra in view of the question under concern in this paper, namely the cut-off of ray trajectories by the instantaneous wave surface.

An alternative statistical formalism can be derived for an arbitrary spectrum in which the statistics of the local positive maxima of the wave surface ("peaks" above mean sea level) are considered. Cartwright and Longuet-Higgins (1956) have presented the cumulative distribution function $F_{Z}(\zeta)$ of the heights of local positive maxima, denoted as $Z$, in random wave groups. According to these authors, the probability that a local positive maximum $\mathrm{Z}$ will exceed a certain height $\zeta$ equals to $1-F_{Z}(\zeta)$, where the $\operatorname{cdf} F_{Z}(\zeta)$ is given by:

$$
\begin{aligned}
F_{Z}(\zeta)= & \frac{2}{1+\sqrt{1-\varepsilon^{2}}}\left[\Phi\left(\frac{\zeta}{\varepsilon \sqrt{m_{0}}}\right)-\frac{1}{2}\left(1-\sqrt{1-\varepsilon^{2}}\right)\right. \\
& \left.-\sqrt{1-\varepsilon^{2}} \Phi\left(\frac{\sqrt{1-\varepsilon^{2}}}{\varepsilon} \frac{\zeta}{\sqrt{m_{0}}}\right) e^{-\frac{1}{2}\left(\frac{\zeta}{\sqrt{m_{0}}}\right)^{2}}\right]
\end{aligned}
$$

for $0 \leq \zeta<\infty$, where $\mathrm{m}_{\mathrm{n}}$ represents the $n$th moment of the frequency spectrum (equation (6)) and where $\varepsilon$ is the bandwidth parameter defined as:

$$
\varepsilon^{2}=1-\frac{m_{2}^{2}}{m_{0} m_{4}}
$$

Furthermore, $\Phi(z)$ in equation (18) represents the cumulative distribution function of the standardized normal probability density:

$$
\Phi(z)=\frac{1}{\sqrt{2 \pi}} \int_{-\infty}^{z} e^{-t^{2} / 2} d t=\frac{1}{2}\left[1+\operatorname{erf}\left(\frac{z}{\sqrt{2}}\right)\right]
$$

where the error function $\operatorname{erf}(z)$ (see, e.g., Abramowitz and Stegun, 1972) has the form:

$$
\operatorname{erf}(z)=\frac{2}{\sqrt{\pi}} \int_{0}^{z} e^{-t^{2}} d t
$$

As can be seen from equation (18), the probability of a local maximum above a given height depends on the sea state through the moments of the frequency spectrum (the variance $\sigma^{2}=m_{0}$ and the bandwidth parameter $\varepsilon$ ). The corresponding probability density function is given by:

$$
\begin{aligned}
f_{Z}(\zeta)= & \frac{2 / \sqrt{m_{0}}}{1+\sqrt{1-\varepsilon^{2}}}\left[\frac{\varepsilon}{\sqrt{2 \pi}} e^{-\frac{1}{2 \varepsilon^{2}}\left(\frac{\zeta}{\sqrt{m_{0}}}\right)^{2}}\right. \\
& \left.+\sqrt{1-\varepsilon^{2}} \frac{\zeta}{\sqrt{m_{0}}} \Phi\left(\frac{\sqrt{1-\varepsilon^{2}}}{\varepsilon} \frac{\zeta}{\sqrt{m_{0}}}\right) e^{-\frac{1}{2}\left(\frac{\zeta}{\sqrt{m_{0}}}\right)^{2}}\right]
\end{aligned}
$$

In two limiting cases, the above probability density function of positive maxima simplifies considerably. In the first place, when $\varepsilon=0$, which represents an asymptotic narrow band process, equation (22) reduces to the well-known Rayleigh probability density function (14) using the dimensionless variable $\xi=\zeta / m_{0}{ }^{1 / 2}$. In the second place, for $\varepsilon=1$, the 
sea surface displacement would represent a wide-band process and the probability density function of local positive maxima can be described in non-dimensional form as:

$$
f(\xi)=\sqrt{\frac{2}{\pi}} e^{-\xi^{2} / 2}
$$

which is a truncated normal (Gaussian) distribution. For ocean waves, the values of the bandwidth parameter $\varepsilon$ usually lie in the range 0.40 to 0.80 , while those for waves in the surf zone fall in the higher range 0.70 to 0.95 .

Having outlined the statistical formalism, we now turn our attention to the frequency spectrum $S(\omega)$. In this first study, we will consider only sea states dominated by the so-called wind-generated waves. For these conditions, many spectral formulation based on empirical and theoretical studies have been published in the literature (for a review, see Donelan et al., 1985; Elfouhaily et al., 1997). In this study we use the Unified spectrum model proposed by Elfouhaily et al. (1997), which is a composite of a long wave spectrum and a short wave spectrum to describe the full range of wave numbers (from the spectral peak to capillary waves). The original formulation has been provided for the so-called omnidirectional wave number spectrum

$$
S(k)=S_{L}+S_{S}
$$

where the subscripts $\mathrm{L}$ and $\mathrm{S}$ indicate the low and high wave numbers or frequencies. In contrast to equation (5), the wave spectrum $\Psi(\boldsymbol{k}, \omega)$ is now integrated with respect to frequency, which yields the wave number spectrum $\Psi_{k}(\mathbf{k})$. When the angular dependence is removed, the omni-directional wave number spectrum is obtained:

$$
S(k)=\int_{-\pi}^{\pi} \Psi_{k}(k, \theta) k \mathrm{~d} k \mathrm{~d} \theta \quad k=|\mathbf{k}|
$$

where $(k, \theta)$ represent polar coordinates in wave number space. Here $\Psi_{\mathrm{k}}$ indicates the wave number spectrum that can be obtained by integrating the wave spectrum $\Psi(\mathbf{k}, \omega)$ with respect to frequency. Details on the exact formulation can be found in the paper by Elfouhaily et al. (1997) and will not be reproduced here.

The Unified spectrum can model a wide range of conditions: from fetch-limited to large fetch values as found for open ocean conditions (the latter due to corrections to the formalism proposed by Donelan et al. (1985)). Furthermore, it was shown that the Unified model correctly reproduces the significant wave height for both developing and fully developed seas. It has also been favorably compared to the measured mean square slopes of the sea surface for both clean and oilslick covered water surfaces. Figure 1 shows the Unified wave spectrum representative of fully developed wind waves for three different values of the wind speed $U_{10}$, and for an inverse wave age value $U_{10} / C_{P}$ of 0.84 corresponding to infinite fetch conditions. Figure 2 shows the total variance and the bandwidth parameter $\varepsilon$ for a wind speed interval between 0 and $25 \mathrm{~m} / \mathrm{s}$. For comparison, results from two other wave spectral models are included in figure 2 . The Pierson-Moskowitz spectrum applies only to fully developed wind-waves, e.g. situations with very large fetch (which corresponds to an inverse wave age $U_{10} / C_{P}$ of approximately 0.84 ). The Donelan et al formalism is a modification of the JONSWAP spectrum (Hasselmann et al. 1973) to describe fetch-limited and fully developed wind-waves. As shown in figure 2, the differences between the predicted total variance and bandwidth are small for low wind speeds, but increase for wind speed greater than about $10 \mathrm{~m} / \mathrm{s}$. For the total variance, the predictions obtained with the Unified spectrum lie between those from the Pierson Moskowitz spectrum and the Donelan et al. spectrum. At high winds speeds, the bandwidth parameter proposed by Donelan et al. (1985) is larger than the values published by Pierson and Moskowitz, whereas the Unified spectrum predicts slightly lower values. 


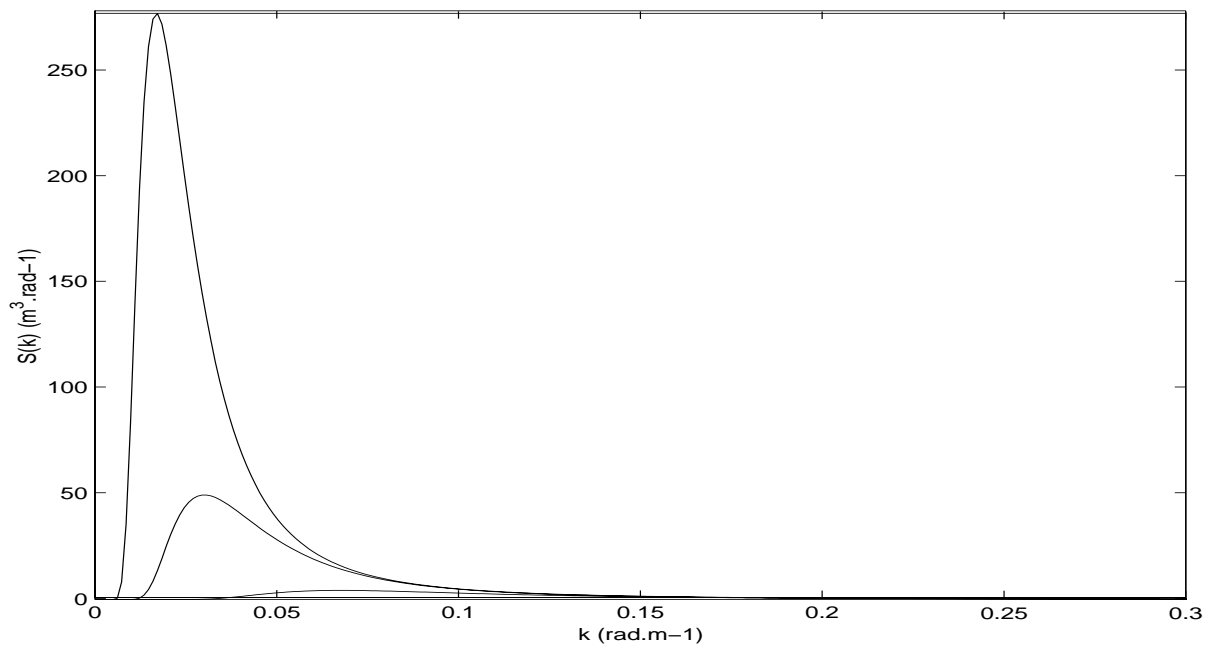

Figure 1: Unified Wave spectrum with $\mathrm{U}_{10} / C_{P}=0.84$ for three different wind speed $U_{10}=10,15,20 \mathrm{~m} / \mathrm{s}$. The peak wave number shifts towards small values while the peak amplitude increases as the wind increases.
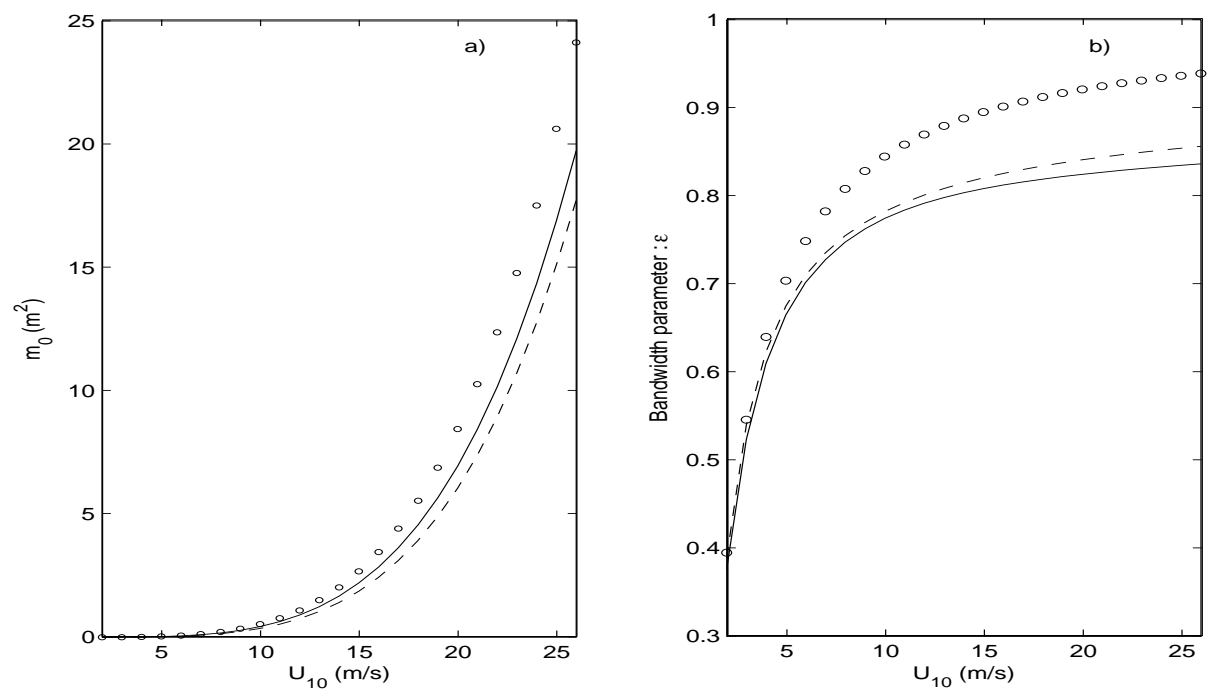

Figure 2: Parameters of the wave spectrum as a function of wind speed with $\mathrm{U}_{10} / C_{P}=0.84$. a) Total variance $m_{0}$ of the wave field; b) Bandwidth parameter $\varepsilon$. (- - -: Pierson-Moskowitz spectrum, o o o: Donelan et al. spectrum, —: Unified spectrum)

\section{NUMERICAL RESULTS}

In a non-refracting and clear atmosphere the maximum detection range of targets over a flat ocean is determined by geometric considerations taking into account the heights of camera and target, the distance between the camera and the target, and the Earth' curvature. However, in the atmospheres generally encountered over marine surfaces, the observation paths of the individual camera pixels, or ray trajectories, are not straight lines but curved. This is due to vertical variations of temperature and humidity, which in turn cause vertical variations in the refractivity of air and thus cause refraction of rays and a change in direction. Figure 3 shows an example of a set of ray trajectories as calculated by EOSTAR. The sensor is placed at 18 meters height in the left of the figure, and each line represents the instantaneous 
field-of-view of an individual sensor pixel. The following atmospheric conditions at a height of $10 \mathrm{~m}$ have been used: air temperature $17 \mathrm{C}$, humidity $80 \%$, pressure $1013 \mathrm{hPa}$, whereas the sea surface temperature was set to $22 \mathrm{C}$.

Depending on the (vertical) slope of the refractivity this can increase or decrease of the maximum detection range for a target at a certain height or the minimum detection height of a target at a certain distance. Upward bending of ray trajectories occur when the slope of the refractivity is negative, thus when the overlaying air is colder than the sea surface. In those cases, the rays initially starting at a smaller elevation angle are stronger refracted upwards than rays starting at a larger elevation angle. As a result, these rays will cross at a certain range (see figure 3 ). When ray crossing occurs, both rays image the same volume and the sensor thus views a secondary image of the target, which is referred to as a mirage. For a given target height, the minimum mirage range (MMR) is the range at which the mirage is first seen. The maximum intervision range (MIVR) is the maximum distance at which the target is in the field-of-view of the sensor. The horizontal mirage interval is the difference between MIVR and MMR. It is indicated in figure 3 by the double-ended horizontal arrow.

The horizontal line at 0.5 meters height in figure 3 represents the cut-off height of the rays. When a ray descends below this height, it is considered to hit the water surface (wave tops). In the simplest approach, EOSTAR places this cut-off height at the crest of the Stokes wave (see section 2). Figure 3 shows that MMR and MIVR are dependent on the cut-off height. The formalism presented in the previous section now allows us to calculate the statistical distribution of wave height, and thus to study the statistical properties of MMR and MIVR.

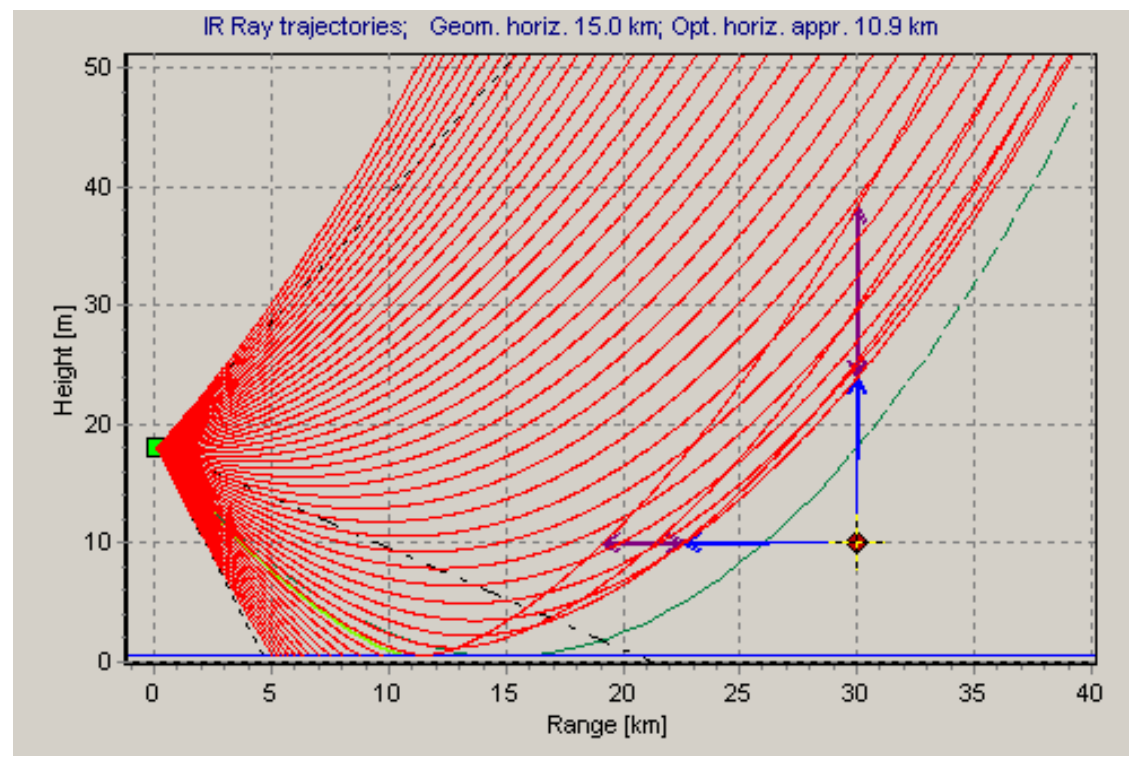

Figure 3: Ray trajectories for a sensor (square symbol on the left axis) at a height of $18 \mathrm{~m}$ above mean sea level under a mirage condition $(\mathrm{Ta}=17 \mathrm{C}, \mathrm{Ts}=22 \mathrm{C}, \mathrm{U}=5 \mathrm{~m} / \mathrm{s}$ at $10 \mathrm{~m})$. The target is represented by the circular symbol in the right hand side of the figure. The single-ended arrows indicate the horizontal and vertical distance between the target and the sensor's field-of-view. The doubleended arrows indicate the mirage interval. The horizontal line at $0.5 \mathrm{~m}$ height represents the mean crest height in this example.

Using the Unified spectrum (representative of wind-generated waves), the statistical distribution of wave heights have been calculated using equations (14) (narrow-band assumption) and (22). For applications in which only the statistics of the heights of the wave-crests are important, the Unified spectrum can be considered in the frequency range $\left[0,6 \omega_{p}\right]$ where the peak frequency is a function of wind speed. Thus, only the long wave spectrum is essential for our application. The results of both methods were almost identical, as was to be expected since the Unified spectrum in the above frequency range can be considered as a narrow-band. More significant differences are expected when mixed seas (windgenerated waves and swell) are considered. 
The statistical results have subsequently been entered in EOSTAR, and for the atmospheric conditions given above, EOSTAR calculated the maximum detection range, the minimum detection height and the width and height intervals of the mirage area. Two wind speeds have been considered: 5 and $20 \mathrm{~m} / \mathrm{s}$.

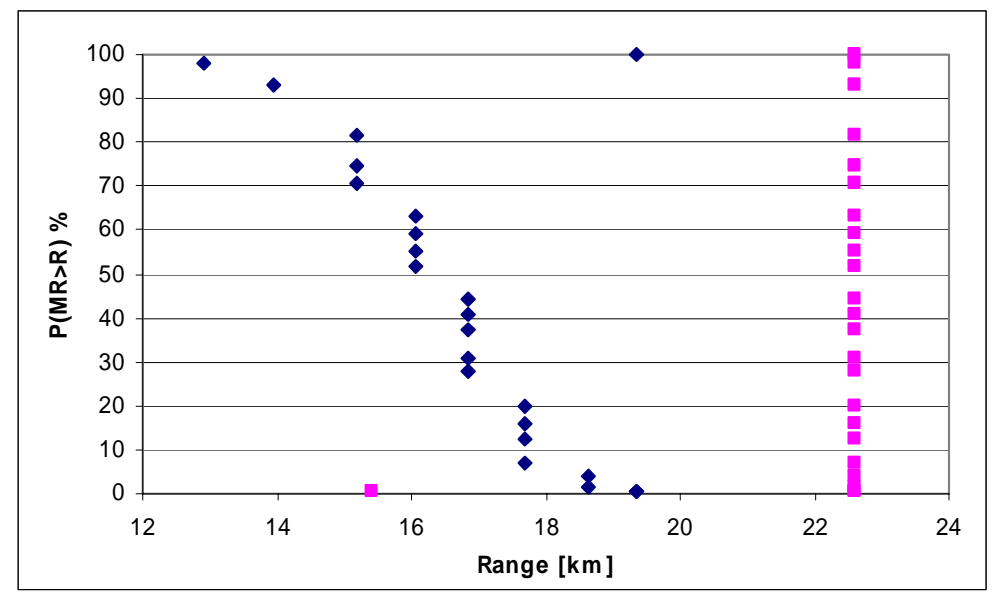

Figure 4: Probability distribution of the maximum detection range (squares) and the minimum mirage range (diamonds) at a wind speed of $5 \mathrm{~m} / \mathrm{s}$, for targets at $10 \mathrm{~m}$ above mean sea level.

First, the results for a wind speed of $5 \mathrm{~m} / \mathrm{s}$ are presented in Figures 4 and 5. The vertical axis in Figure 4 represents the probability that the MMR or MIVR for a target at $10 \mathrm{~m}$ height is larger than a certain value. Figure 4 shows that he variation of the maximum intervision range with wave height is negligible, but that the MMR is strongly dependent on wave height. For a flat surface at mean sea level, the MMR is at about $13 \mathrm{~km}$. Since all waves will be higher, the probability that the MMR exceeds $13 \mathrm{~km}$ is $100 \%$. Since the probability of very high waves decreases, the probability of having a large MMR also decreases. The figure shows that the probability of having MMR $>18.5 \mathrm{~km}$ is almost zero for the current wave conditions. The mean value of MMR is approximately $16 \mathrm{~km}$ and the mean mirage interval thus extends from 16.0 to $22.5 \mathrm{~km}$. The figure further shows that there is a fair chance of having variations in MMR of the order of a kilometer due to fluctuations in the instantaneous wave surface.

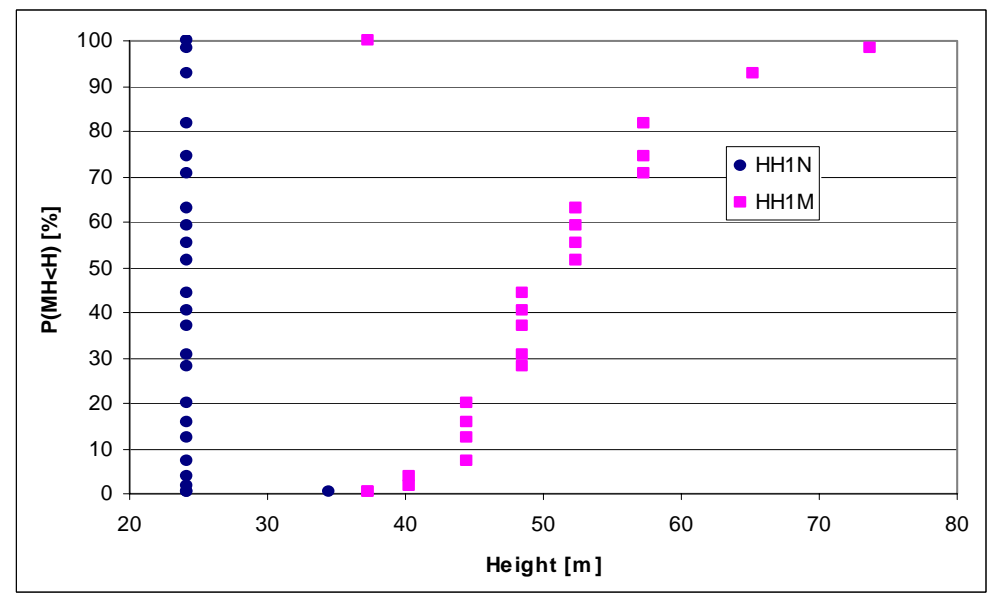

Figure 5: Probability distribution of the minimum intervision height (diamonds) and maximum mirage height (squares) for a target at a range of $30 \mathrm{~km}$ from the sensor and at a wind speed of $5 \mathrm{~m} / \mathrm{s}$. 
Figure 5 repeats this analysis for the vertical. The vertical axis in Figure 5 now represents the probability that a target at a distance of $30 \mathrm{~km}$ will be imaged as a mirage at a height lower than a given height. Thus the probability is $100 \%$ that the mirage interval starts lower than $70 \mathrm{~m}$ and the probability is almost $0 \%$ that the interval starts lower than about $35 \mathrm{~m}$. The $50 \%$ probability is that the interval starts at 50 meters. The minimum intervision height is almost independent of the wave surface, and is located at 23 meters.

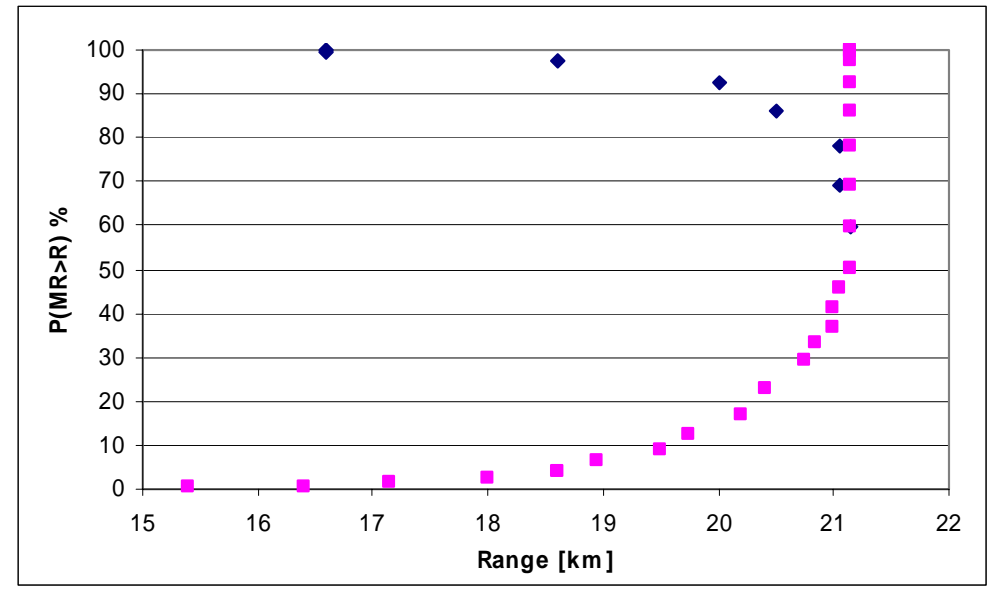

Figure 6: Probability distribution of the maximum intervision range (squares) and the minimum mirage range interval (diamonds) for targets at $10 \mathrm{~m}$ above mean sea level and at a wind speed of $20 \mathrm{~m} / \mathrm{s}$.

Figures 6 and 7 present the results for a wind speed of $20 \mathrm{~m} / \mathrm{s}$. Figure 6 shows that in this case the MIVR is also dependent on wave height. On the average, the MIVR amounts to $21 \mathrm{~km}$, but there is a chance of about $10 \%$ that the view of the sensor pixel is blocked by a high wave reducing the MIVR to less than $19.5 \mathrm{~km}$. Figure 6 further shows that the higher waves may cut so many rays that no mirage is observed at all. If present, the mirage occurs only at ranges between $16 \mathrm{~km}$ and $21 \mathrm{~km}$, but with a probability of less than $30 \%$. This shows the importance of the present statistical analysis. A simpler version of EOSTAR would have considered a fixed value for the wave height (corresponding to 50 $\%$ probability in figure 6) and the conclusion would thus have been that there is no mirage zone for a target at 10 meters above mean sea level. With the present statistical analysis, we are able to state that there is still a $30 \%$ chance of observing mirages under these conditions.

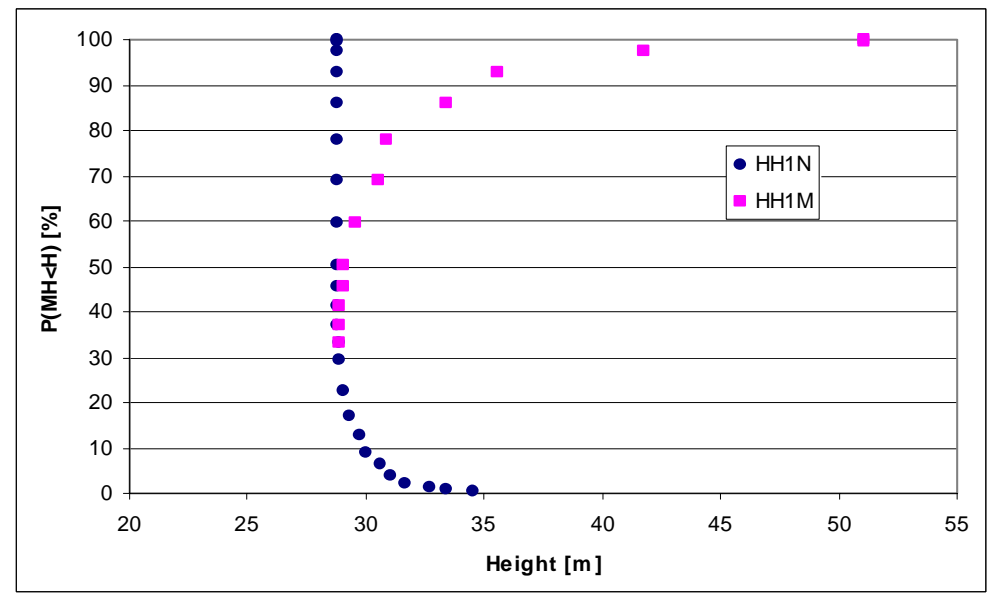

Figure 7: Probability distribution of the minimum intervision height (diamonds) and the maximum mirage height (squares) at a range of $30 \mathrm{~km}$ from the sensor and at a wind speed of $20 \mathrm{~m} / \mathrm{s}$. 
Figure 7 shows the results in the vertical plane for a target at $30 \mathrm{~km}$. The figure shows that the target is always in view of the sensor when it is located at a height of 35 meters or more. There is no probability of having the target in view when it is located below $28 \mathrm{~m}$ height. The mirage zone may extend between 28 and $51 \mathrm{~m}$ height, but there is only some $30 \%$ chance of observing a mirage.

\section{CONCLUSION}

We have presented two different approaches to describe the statistical properties of the wave spectrum, in particular the probability that the wave surface exceeds a certain height above mean sea level. Both approaches are based on a given wave spectrum distribution of the wave energy, which characterizes the sea state. The first approach is based on the narrow-band assumption, for which the probability of occurrence of large wave crest depends only on the sea state via the variance $m_{0}$ of the wave field. The second approach is based on the theory of random wave groups. It gives a probability of occurrence that depends not only on the variance $m_{0}$ but also on the bandwidth parameter $\varepsilon$. For the present study using the Unified spectrum for wind-generated waves there is no preference to use either of the models, although the latter model has a wider range of applicability because it does not assume any narrow-banded spectrum.

It has been shown that for a given condition the maximum detection range, the minimum detection height and the magnitude of the mirage interval are not fixed but are statistically distributed. This distribution can be quantified and is related to the statistical distribution of wave-crest heights. The statistical distribution of the wave-crest heights induces strong variations in the occurrence and size of mirage zones. In turn, and depending on sensor resolution, this may induce strong variations in signal strength of small targets in the vicinity of their maximum intervision range. Such signal variations should be distinguished from atmospheric turbulence, causing scintillation and blur.

Wave statistics not only impact on geometrical effects but also on effects relating the exchange of momentum, heat and water vapor, and thus on the turbulent refractive properties of the marine surface layer. It should be emphasized that the short wave spectrum is of crucial importance for accurate parameterization of these exchange processes in the lowest meters of the marine atmospheric surface layer. Recently, the Unified spectrum has been used in a coupled sea surfaceatmosphere model (see Makin and Kudryatsev, 1999) to study the direct and indirect effects of the wave-induced motion in the atmospheric surface layer. These effects are thought to decay rapidly with height, although the actual variation with height and the dependence of the air-sea fluxes with sea state are still uncertain. This is of particular importance to the description of microwave or infrared propagation, because they can modify the refractivity index in the lowest meters of the atmospheric surface layer. These issues are subject for future work.

\section{REFERENCES}

Abramowitz, M. and Stegun, I. A., Handbook of mathematical functions, Dover Publ., New York (1972).

Cartwright, D. E.and Longuet-Higgins, M. S., The statistical distribution of the maxima of a random function, Proc. Roy. Soc. London., Ser. A, 237, pp. 212-232 (1956).

Donelan M. A., Hamilton J. and Hui W. H., Directional spectra of wind generated waves, Philos. Trans. R. Soc. London., Ser. A, 315, pp. 509-562 (1985).

Elfouhaily T., Chapron B., Katsaros K. and Vandermark D., A unified directional spectrum for long and short winddriven waves, J. Geophys. Res., 102 (C7), pp. 15 781-15796 (1997).

Geernaert, G. L., 1990, Mechanics of ocean surface waves, In: Surface waves and fluxes I, 209-246, G.L Geernaert and W.J. Plant (eds), Kluwer Academic Press (1990).

Hasselmann K. et al., Measurements of wind-wave growth and swell decay during the Joint North Sea Wave Project (JONSWAP), Dtsch. Hydrogr. Inst., Hamburg (1973). 
Kinsman, B., Wind waves, Prentice Hall, Englewood Cliffs, (1965).

Kunz G. J., Degache, M. A. C., Moerman M. M., van Eijk, A. M. J. Neele, F.P. Doss-Hammel S. M. and Tsintikidis D., Status and developments in EOSTAR, a model to predict IR sensor performance in the marine environment, Gonglewski J. D. and Stein K., Eds. , Proc. SPIE Vol. 5572, pp. 101-111 (2004)

Longuet-Higgins, M. S., 1957, The statistical analysis of a random moving surface, Proc. Roy. Soc. London., Ser. A, 349, pp. 321-387 (1957).

Makin, V. K. and Kudryatsev V. N., 1999, Coupled sea surface-atmosphere model 1. Wind over waves coupling, J. Geophys. Res., 104, pp. $7625-7639$ (1999).

Neele, F.P., Infrared ship signature prediction, model validation and sky radiance, to be published in: Targets and Backgrounds XI: Characterization and Representation, SPIE Proc., vol. 5811 (2005).

Ochi, M. K., Ocean waves: the stochastic approach, Camb. Univ. Press, Cambridge (1990).

Rice, S. O., Mathematical analysis of random noise, Bell. Syst. Tech. J., 23, pp. 282- 332 (1944).

Schwering P.B.W., Marine infrared background clutter. SPIE Proceedings 2742, 255-266, (1996).

Van Eijk A.M.J., Cohen L.H., Navarro L.J. and De Leeuw G., Near-surface aerosol transmission in the marine environment. SPIE Proceedings 4884, 160-169, (2002). 